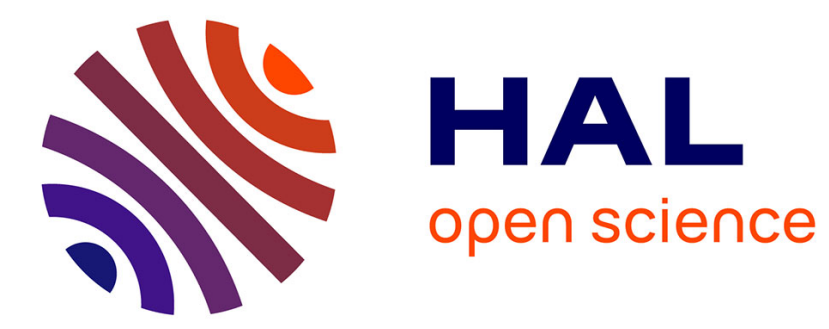

\title{
En route vers le Petawatt
}

P. Maine, D. Strickland, P. Bado, M. Pessot, G. Mourou

\section{To cite this version:}

P. Maine, D. Strickland, P. Bado, M. Pessot, G. Mourou. En route vers le Petawatt. Revue de Physique Appliquée, 1987, 22 (12), pp.1657-1663. 10.1051/rphysap:0198700220120165700 . jpa-00245725

\section{HAL Id: jpa-00245725 https://hal.science/jpa-00245725}

Submitted on 1 Jan 1987

HAL is a multi-disciplinary open access archive for the deposit and dissemination of scientific research documents, whether they are published or not. The documents may come from teaching and research institutions in France or abroad, or from public or private research centers.
L'archive ouverte pluridisciplinaire HAL, est destinée au dépôt et à la diffusion de documents scientifiques de niveau recherche, publiés ou non, émanant des établissements d'enseignement et de recherche français ou étrangers, des laboratoires publics ou privés. 


\title{
En route vers le Petawatt
}

\author{
P. Maine, D. Strickland, P. Bado, M. Pessot et G. Mourou \\ Laboratory for laser energetics, Ultrafast Science Center, University of Rochester, 250 East River Road, \\ Rochester, New York 14623-1299, U.S.A.
}

(Reçu le 27 juin 1987, accepté le 24 août 1987)

\begin{abstract}
Résumé. - Des impulsions d'une durée de 1 ps ont été amplifiées jusqu'au niveau du terawatt par un système très compact à verre dopé au néodyme basé sur l'utilisation de la technique d'amplification des impulsions à dérive de fréquence. La divergence du faisceau est égale à deux fois la limite de la diffraction, donnant à cette source une brillance de $2 \times 10^{+18} \mathrm{~W} /\left(\mathrm{cm}^{2} . \mathrm{sr}\right)$, ce qui constitue la brillance la plus élevée jamais reportée à ce jour. La technique d'amplification d'impulsions à dérive de fréquence rend possible l'extraction de l'énergie de manière efficace dans des systèmes d'amplification extrêmement compacts. L'amplification des impulsions à dérive de fréquence a été démontrée sur neuf ordres de grandeur, du nanojoule jusqu'au niveau du joule. En utilisant une chaîne d'amplification à verre au néodyme de grandes dimensions, il devrait être possible d'étendre cette technique à l'amplification d'impulsions de cent femtosecondes jusqu'au niveau d'une centaine de joules, générant ainsi une puissance instantanée de l'ordre du pétawatt. La focalisation ultérieure de ces impulsions pourrait alors donner naissance à des intensités de l'ordre de $10^{23} \mathrm{~W} / \mathrm{cm}^{2}, 5$ ordres de grandeurs audessus des performances actuelles.
\end{abstract}

\begin{abstract}
Single picosecond pulses have been amplified to the terawatt level by a table-top size Nd :glass amplifier by using the technique of chirped pulse amplification (CPA). The divergence of the beam is twice the diffraction limit making the brightness of this source equal to $\sim 2 \times 10^{+18} \mathrm{~W} /(\mathrm{cm}$. sr), which is the highest brightness ever reported. The technique of chirped pulse amplification alows the efficient energy extraction from extremely compact amplifier systems. Amplification of chirped pulses over nine orders of magnitude, i.e., from nanojoule to the joule level, has been demonstrated. By using a large scale $\mathrm{Nd}$ :glass amplifier system, it should be possible to extend the technique of CPA to the amplification of hundred femtosecond pulses to the hundred joule level leading to petawatt power pulses. These power pulses once focused could produce intensity in the range of $10^{23} \mathrm{~W} / \mathrm{cm}^{2}$, five order of magnitude over the present state of the art.
\end{abstract}

\section{L'amplification d'impulsions courtes.}

Un milieu amplificateur, afin d'amplifier des impulsions courtes, a trois conditions à satisfaire. Premièrement, il doit posséder une bande passante suffisament large pour amplifier la totalité du spectre de l'impulsion. Deuxièmement, dans le but d'extraire l'énergie stockée dans l'amplificateur de manière efficace, il est nécessaire que la fluence de l'impulsion soit proche de la fluence de saturation du milieu, $F_{\mathrm{s}}=h v / \sigma, \sigma$ étant la section efficace d'émission stimulée. Enfin, l'intensité de l'impulsion se propageant dans l'amplificateur doit rester au-dessous d'une valeur critique, valeur pour laquelle les effets non linéaires ne peuvent plus être négligés, et induisent une distorsion des profils spatial et temporel de l'impulsion. L'intégration de l'indice non linéaire le long du trajet optique est donnée par l'intégrale $B[1]$ :

$$
B=\frac{2 \pi}{\lambda} \int \frac{\Delta n}{n} \mathrm{~d} l=\frac{2 \pi}{\lambda} n_{2} \int_{0}^{L} I(z) \mathrm{d} z
$$

L'intégrale $B$ est la mesure du déphasage induit par les effets non linéaires entre un faisceau intense et un faisceau de faible intensité. L'intensité critique est définie comme étant celle correspondant à une valeur de $B$ égale à cinq. Au-dessus de cette valeur, les fréquences spatiales élevées sont amplifiées de manière préférentielle et un filtrage spatial est nécessaire. Dans le cas des colorants et des lasers solides, l'intensité critique est de l'ordre de $10 \mathrm{GW} / \mathrm{cm}^{2}$.

Les impulsions courtes sont communément ampli- 
fiées dans des amplificateurs à colorant ou des lasers excimères. Ces milieux, qui ont une bande passante de l'ordre de $20 \mathrm{~nm}$, sont capables d'assumer l'amplification d'impulsions d'une durée à peu-près égale à 30 fs. Néanmoins, la fluence de saturation de ces milieux n'est que de l'ordre d'un millijoule par centimètre carré. De ce fait, des impulsions de $100 \mathrm{fs}$ peuvent être amplifiées jusqu'à saturation sans que leur intensité ne dépasse la valeur critique. Pour cette raison les colorants sont bien adaptés à l'amplification d'impulsions courtes jusqu'au niveau du millijoule. Des énergies plus élevées peuvent alors être obtenues en amplifiant l'impulsion dans des excimères, au prix d'une importante augmentation de la section de l'amplificateur [2].

Comme nous venons de le voir, une faible fluence de saturation, associée à un temps de stockage de seulement quelques nanosecondes, fait des colorants et des excimeres des milieux amplificateurs loin d'être idéaux. Le choix d'un milieu possédant une fluence de saturation mille fois plus élevée peut permettre la génération d'énergies au niveau du joule avec un système compact. Les milieux solides dopés au néodyme, au chrome, ou au titane ont une fluence de saturation de l'ordre de $5 \mathrm{~J} / \mathrm{cm}^{2}$. De plus, ils acceptent des concentrations en ions actifs supérieure à $10^{20}$ atomes par centimètre cube. Les cristaux dopés au chrome ont déjà prouvé leur capacité à « laser " de 700 à $1100 \mathrm{~nm}$ avec de larges bandes passantes ; l'un d'eux, l'alexandrite, qui couvre la bande $700-800 \mathrm{~nm}$, a déjà produit des puissances moyennes de l'ordre de $100 \mathrm{~W}$ [3]. Un autre milieu très prometteur est le $\mathrm{Ti}$ :sapphire, qui à lui seul peut amplifier les longueurs d'ondes comprises entre 700 et $1000 \mathrm{~nm}$. Le verre au néodyme est un milieu amplificateur aujourd'hui largement répandu qui, avec une bande passante de $20 \mathrm{~nm}$, peut amplifier des impulsions de $100 \mathrm{fs}$. Néanmoins, l'amplification d'impulsions de 1 ps jusqu'à saturation dans ces milieux conduirait à des densités de puissance supérieures à $1 \mathrm{TW} / \mathrm{cm}^{2}$, ce qui est de loin au-dessus de la valeur critique. De ce fait, la grande quantité d'énergie qui est stockée dans l'amplificateur ne peut pas être extraite avec une impulsion courte. C'est la raison pour laquelle les amplificateurs solides sont largement utilisés pour l'amplification d'impulsions relativement longues (ns), alors qu'ils sont rarement employés pour l'amplification d'impulsions picosecondes.

Une nouvelle technique est donc nécessaire pour maintenir de faibles puissances crête dans l'amplificateur. Il y a de cela plus de quarante ans, des scientifiques travaillant dans le domaine du radar ont surmonté une telle limitation en imposant sur l'impulsion une dérive de la fréquence instantanée («chirp») et en l'étirant au moyen d'une ligne dispersive avant de l'amplifier [4]. L'amplification d'impulsions rendues ainsi plus longues permettait l'extraction d'énergies plus élevées. L'écho radar était alors couplé dans un filtre adapté qui permettait la synchronisation de toutes les fréquences de telle sorte que la durée de l'impulsion soit approximativement égale à $1 / \Delta f$, où $\Delta f$ est la bande passante de la dérive de fréquence. Nous avons développé une technique analogue pour l'amplification d'impulsions courtes dans le domaine optique. Le principe de cette technique d'amplification d'impulsions possédant une dérive de fréquence ( CPA » = Chirped Pulse Amplification) est décrit par la figure 1: une impulsion optique brève subit tout d'abord une dérive en fréquence et est ainsi étirée, ce qui permet de l'amplifier jusqu'à saturation tout en maintenant sa puissance instantanée à un faible niveau. Une fois amplifiée, un compresseur optique est utilisé pour ramener l'impulsion à sa durée initiale, produisant ainsi une impulsion à la fois brève et très énergétique.

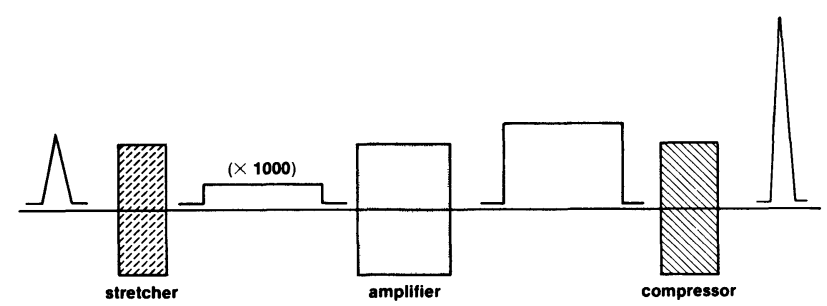

Fig. 1. - Technique d'amplification des impulsions à dérive de fréquence.

[Chirped pulse amplification technique.]

Deux variantes de la technique «CPA » ont été démontrées. La première est basée sur la compression d'impulsions à l'aide de fibres et de réseaux et sera expliquée dans la section 2, alors que la deuxième utilise une paire de réseaux à la fois pour l'allongement et la compression et sera discutée dans la section 3 .

\section{Génération d'impulsions de 0,5 TW en 1 ps.}

Ainsi que nous l'avons reporté précédemment, nous avons démontré l'amplification d'impulsions à dérive de fréquence utilisant un système de compression à fibre et réseaux $[5,6]$. Le système original était composé d'un oscillateur Nd : YAG et d'amplificateurs à verre silicate dopés au néodyme et amplifiait des impulsions de 2 ps jusqu'à $100 \mathrm{~mJ}$. Nous utilisons maintenant un oscillateur $\mathrm{Nd}: \mathrm{YLF}$ à verrouillage de modes pompé en continu qui génère des impulsions de 55 ps et permet ainsi d'atteindre des durées d'impulsions plus courtes après compression [7]. Nd: YLF présente aussi l'intérêt d'opérer à une longueur d'onde centrée sur la bande de gain des verres phosphates qui ont de meilleures caractéristiques thermiques que les silicates. Le système actuel 
génère des impulsions de puissance crête $0,5 \mathrm{TW}$ en 1 ps.

Un diagramme du système laser est montré en figure 2. Un oscillateur ND: YLF à verrouillage de modes génère un train d'impulsions de 55 ps à un taux de répétition de $100 \mathrm{MHz}$, la longueur d'onde d'émission étant de $1,053 \mu \mathrm{m}$. Ces impulsions sont

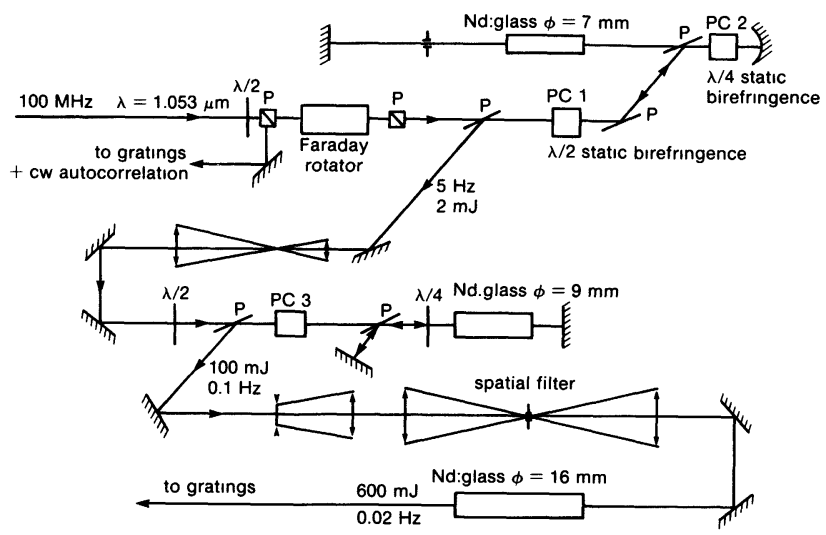

Fig. 2. - Description du système laser actuel.

[Diagram of the current laser system.]

ensuite couplées dans une fibre optique monomode d'une longueur de $1,3 \mathrm{~km}$, dont le cœur a un diamètre de $9 \mu \mathrm{m}$, dans laquelle les effets combinés de l'automodulation de phase et de la dispersion de vitesse de groupe vont générer une impulsion possédant une dérive en fréquence extrêmement linéaire de largeur spectrale $3,5 \mathrm{~nm}$ et de durée $300 \mathrm{ps}$ [8]. A ce niveau, la compression de ces impulsions peut être réalisée à l'aide d'une paire de réseaux utilisée en double passage [9], produisant des impulsions dont la durée à mi-hauteur est de $1 \mathrm{ps}$. La trace d'autocorrélation de ces impulsions est montrée en figure 3. Avec la technique de CPA les impulsions possédant un «chirp» (300 ps) sont tout d'abord amplifiées, puis compressées. De cette manière, l'énergie que l'on peut extraire du milieu amplificateur est 300 fois plus élevée que si l'on amplifiait directement une impulsion de 1 ps.

Les impulsions possédant un « chirp » sont amplifiées dans un verre phosphate au néodyme athermal (Kigre Q98) dont le maximum de fluorescence est à $1,053 \mu \mathrm{m}$. Ce verre a une bande passante de $21 \mathrm{~nm}$ qui devrait permettre l'amplification d'impulsions de 100 fs. Des énergies jusqu'au niveau du joule sont obtenues en utilisant trois amplificateurs à barreau de verre pompés par lampes flash. Un schéma de l'étage d'amplification est donné en figure 4. Un amplificateur régénératif est tout d'abord utilisé. L'impulsion effectue à peu près 100 aller-retour avant d'atteindre la saturation dans une cavité linéaire qui contient un barreau de $7 \mathrm{~mm}$ de diamètre. Elle est alors éjectée de la cavité avec une

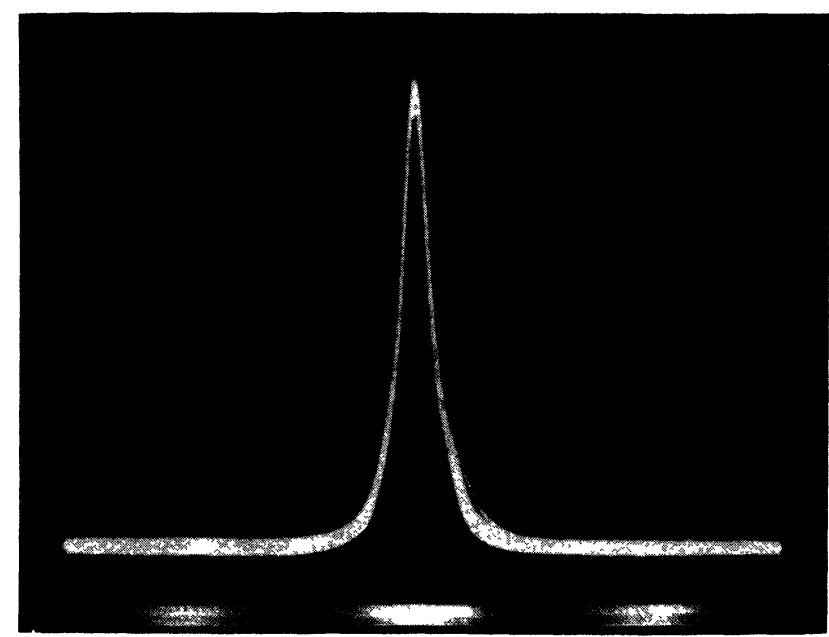

Fig. 3. - Autocorrélation des impulsions du Nd: YLF après compression montrant une durée d'impulsion de 1 ps à mi-hauteur (pour un profil gaussien).

[Autocorrelation trace of the compressed Nd : YLF showing a pulse width of $1 \mathrm{ps}$ assuming Gaussian profile.]

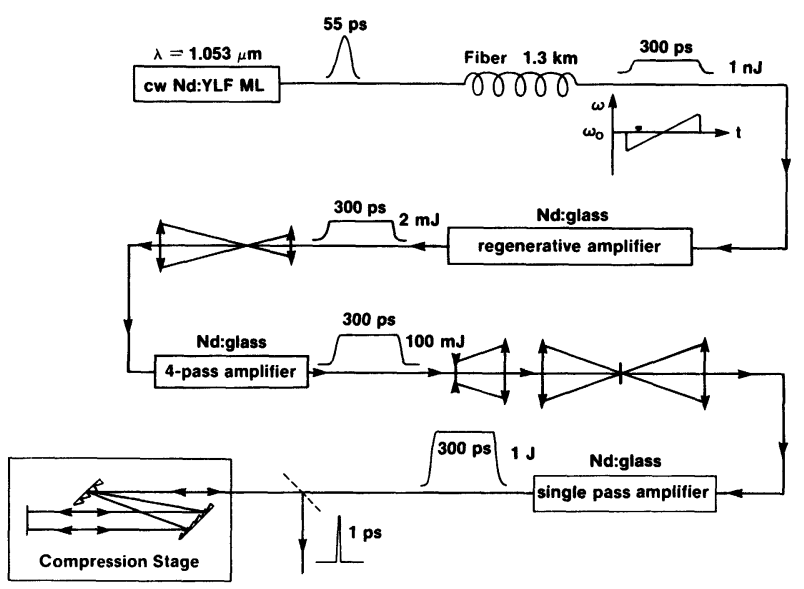

Fig. 4. - Schéma de l'étage d'amplification.

[Schematic of the amplification stage.]

énergie de $2 \mathrm{~mJ}, 6$ ordres de grandeur au-dessus de l'énergie initiale (nJ). Après avoir augmenté le diamètre du faisceau, l'impulsion est amplifiée jusqu'à $100 \mathrm{~mJ}$ après 4 passages dans un barreau de $9 \mathrm{~mm}$ de diamètre. Le faisceau subit alors de nouveau une augmentation de diamètre, puis un filtrage spatial, avant d'être amplifié dans le troisième étage. Un simple passage dans un barreau de $16 \mathrm{~mm}$ de diamètre amène l'énergie au niveau du joule.

Le compresseur est constitué de deux réseaux holographiques à couche d'or (1 700 traits par mm) qui sont utilisés au voisinage de l'incidence de Littrow $\left(\sim 65^{\circ}\right)$. L'efficacité de diffraction en simple passage pour la paire de réseaux est de $90 \%$ pour la lumière polarisée dans le plan d'incidence. Afin d'opérer au-dessous du seuil de dommage des 
réseaux $\left(100 \mathrm{~mJ} / \mathrm{cm}^{2}\right)$, nous avons décidé de limiter la fluence incidente sur les réseaux à $20 \mathrm{~mJ} / \mathrm{cm}^{2}$, ce qui, compte tenu des dimensions de nos réseaux actuels $\left(7 \times 7 \mathrm{~cm}^{2}\right)$, permet la compression d'impulsions de $500 \mathrm{~mJ}$, à la condition d'opérer en simple passage sur les réseaux.

La durée de l'impulsion amplifiée a été mesurée à l'aide d'un autocorrélateur monocoup dont le fonctionnement est basé sur la génération non collinéaire de la deuxième harmonique [10]. Un schéma de principe de l'autocorrélateur est donné en figure 5, et le résultat de la mesure est montré en figure 6. L'autocorrélation a une largeur à mi-hauteur de 1,4 ps, correspondant à une durée d'impulsion de $1 \mathrm{ps}$ pour un profil gaussien. La puissance instantanée de l'impulsion à la sortie du système est donc de $0,5 \mathrm{TW}$.

Nous avons récemment mesuré la divergence du faisceau à la sortie du compresseur, et ceci pour des niveaux d'amplification de $1 \mathrm{~mJ}$ et $250 \mathrm{~mJ}$. La mesure a été effectuée en mesurant le diamètre de la tâche focale au foyer d'une lentille $(f=5 \mathrm{~m})$ à l'aide d'une barrette de diodes $(25 \mu \mathrm{m}$ de résolution). Les mesures montrent que l'impulsion de $1 \mathrm{~mJ}, 1 \mathrm{ps}$ a une divergence égale à 1,4 fois la limite de la diffraction, rendant possible des intensités supérieures à $10^{16} \mathrm{~W} / \mathrm{cm}^{2}$ avec un objectif ouvert à $f / 1$. Au niveau d'amplification de $250 \mathrm{~mJ}$, la divergence du faisceau est mesurée comme étant 2,2 fois la limite de la diffraction. Cela conduira, lorsque focalisé avec un objectif ouvert à $f / 1$, à des intensités supérieures à $10^{18} \mathrm{~W} / \mathrm{cm}^{2}$. Nous pensons que la différence entre les deux mesures est principalement due à la défocalisation induite par l'effet de lentille
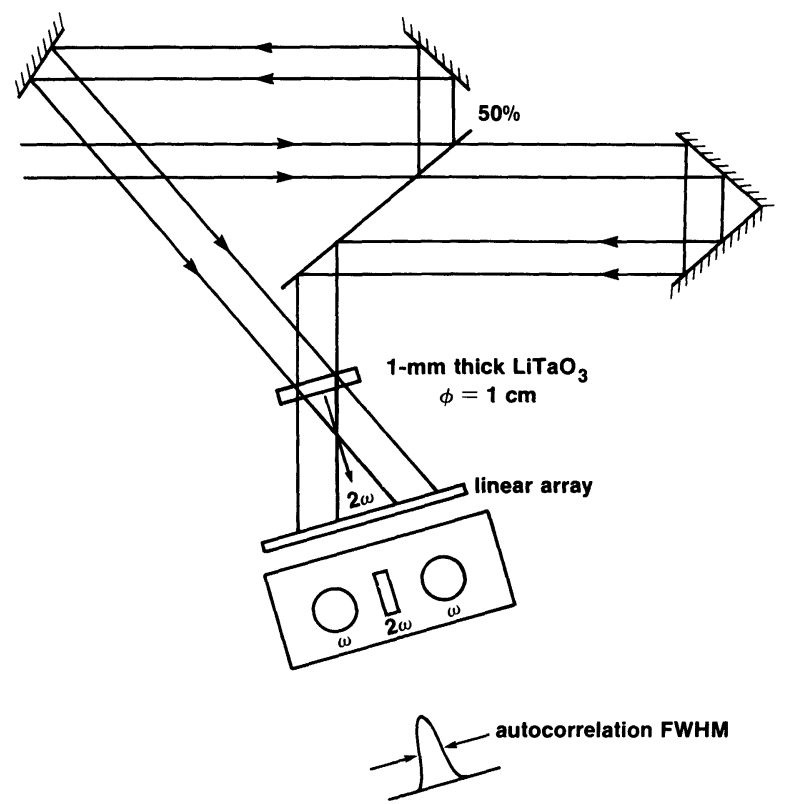

Fig. 5. - Autocorrélateur monocoup.

[Single shot autocorrelator.]

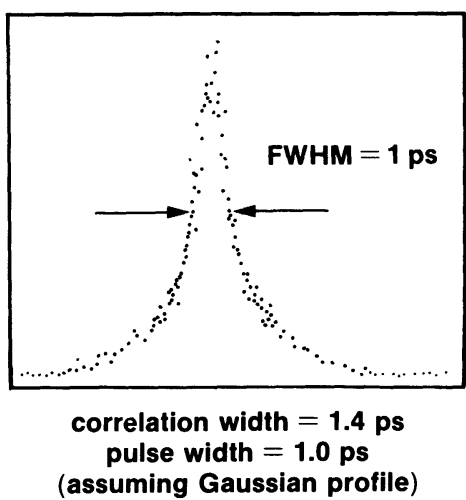

Fig. 6. - Autocorrélation de l'impulsion compressée de $500 \mathrm{~mJ}$ montrant une durée d'impulsion à mi-hauteur de 1 ps (pour un profil gaussien).

[Autocorrelation trace of the $500 \mathrm{~mJ}$ pule showing a pulsewidth of 1 ps assuming a Gaussian profile.]

thermique présent dans le barreau du dernier amplificateur au moment du pompage, et pourra être compensé ultérieurement par une relocalisation du plan image.

A notre connaissance, ceci représente la brillance la plus élevée jamais atteinte. Le système nécessaire à l'obtention d'un tel résultat est remarquablement compact : la totalité de l'étage d'amplification est contenue sur une table de $1,2 \times 2,4 \mathrm{~m}^{2}$. De plus, une optimisation du système, incluant un allongement de

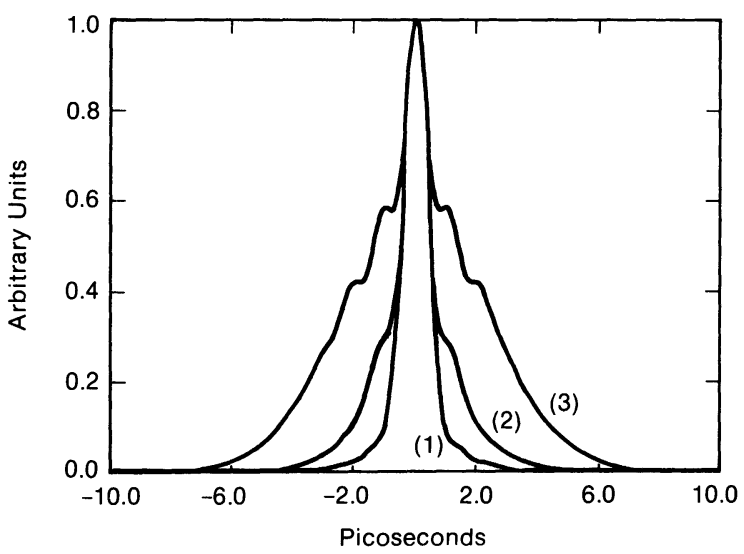

Fig. 7. - Autocorrélations simulées pour une impulsion compressée possédant une bande passante de $3,5 \mathrm{~nm}$ : (1) en l'absence de délai quadratique ; (2) avec le délai quadratique correspondant à une impulsion à «chirp » de 300 ps ; (3) avec le délai quadratique correspondant à une impulsion à «chirp » de 600 ps.

[Autocorrelation of compressed pulses for a $35 \AA$ spectrum : (1) without quadratic delay; (2) with quadratic delay for a 300 ps chirped pulse ; (3) with quadratic delay for a $600 \mathrm{ps}$ chirped pulse.] 
l'impulsion à dérive de fréquence (1 ns) et l'obtention de réseaux de dimensions supérieures, devrait permettre l'extraction d'énergies de l'ordre de 3-4 J du dernier amplificateur. Ces modifications conduiront à une puissance crêtre de l'ordre de $4 \mathrm{TW}$, rendant possibles des intensités de l'ordre de $3 \times 10^{19} \mathrm{~W} / \mathrm{cm}^{2}$.

\section{Vers des puissances plus élevées.}

Afin d'exploiter parfaitement les possibilités offertes par la technique de CPA il est souhaitable que le rapport des durées des impulsions à dérive de fréquence et des impulsions compressées soit le plus grand possible. Or l'utilisation de la technique de compression à fibre et à réseaux impose certaines limitations sur ce rapport. Une valeur de 300 semble être la limite pour les compressions à un seul étage utilisant des oscillateurs Nd: YLF ou Nd: YAG. Cette différence est due à la présence de termes non linéaires dans l'expression décrivant le délai de vitesse de groupe $T$ engendré par une paire de réseaux en fonction de la longueur d'onde. En effet, la compensation de la dérive de fréquence induite dans la fibre par la dispersion de vitesse de groupe produite par une paire de réseaux n'est possible qu'au premier ordre.

Le délai de vitesse de groupe induit par la paire de réseaux peut s'écrire au deuxième ordre [11]:

$$
\begin{aligned}
\tau\left(\lambda_{0}+\Delta \lambda\right)= & \tau_{0}+2 \frac{\lambda_{0}}{c} L \frac{m^{2}}{a^{2} \cos ^{3} \theta} \Delta \lambda+ \\
& +\frac{3 L m^{2}}{c a^{2} \cos ^{3} \theta}\left(1+\frac{\lambda_{0} m \sin \theta}{a \cos ^{2} \theta}\right)(\Delta \lambda)^{2} \\
& =\tau_{0}+\tau_{1}+\tau_{2}
\end{aligned}
$$

où $\lambda=\lambda_{0}+\Delta \lambda$ est la longueur d'onde, $\lambda_{0}$ la longueur d'onde centrale de l'impulsion, $c$ la vitesse de la lumière, $L$ la distance séparant les plans contenant les deux réseaux, $m$ l'ordre de diffraction, $a$ la distance entre deux traits du réseau, et $\theta$ l'angle diffracté pour $\lambda_{0}$. Une impulsion possédant un «chirp» parfaitement linéaire peut alors être compressée en ajustant les paramètres de la paire de réseaux de manière à annuler $\tau_{1}$, mais l'impulsion possède alors une dérive de fréquence résiduelle décrite par $\tau_{2}$. Nous avons simulé la fonction d'autocorrélation ainsi que le profil temporel d'une telle impulsion pour des paramètres appropriés à notre système. La figure 8 montre la déformation de la fonction d'autocorrélation lorsque, pour une largeur de spectre constante $(3,5 \mathrm{~nm})$, on augmente la durée de l'impulsion à dérive de fréquence. La distance entre les réseaux est choisie de manière à annuler $\tau_{1}$. L'effet réel de $\tau_{2}$ sur l'impulsion est pire que l'autocorrélation ne le laisse supposer, ainsi que le

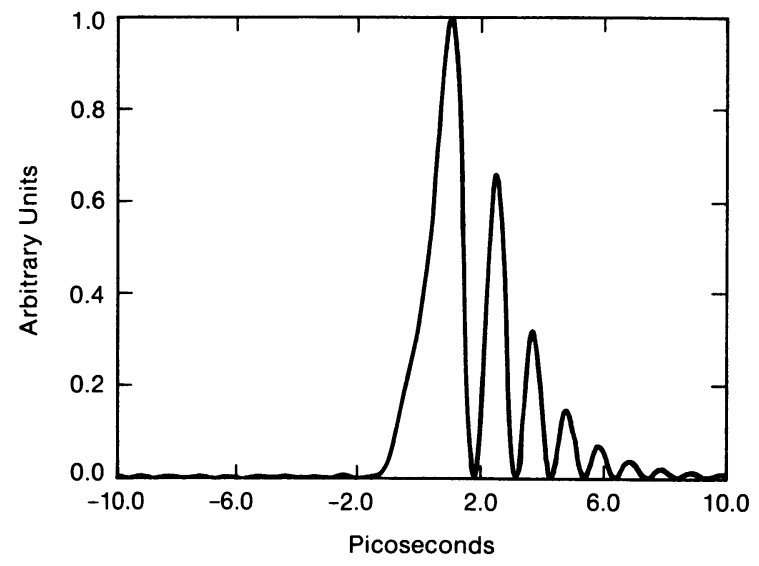

Fig. 8. - Profil temporel résultant de la compression d'une impulsion de 600 ps.

[Temporal profile resulting of the compression of a $600 \mathrm{ps}$ chirped pulse.]

montre le profil temporel de la figure 9. Les battements entre les fréquences symétriques par rapport à la fréquence centrale induits par le délai quadratique modulent très fortement l'impulsion. Des mesures effectuées avec un oscillateur Nd : YAG à verrouillage de modes couplé dans une fibre de $2,4 \mathrm{~km}$ de long illustrent l'importance du terme quadratique : l'autocorrélation des impulsions résultant de la compression d'un «chirp» d'une largeur spectrale de $4 \mathrm{~nm}$ indique une durée d'impulsion à mi-hauteur de 1,2 ps, mais possède un large piedestal (Fig. 9). L'insertion dans le faisceau d'un filtre interférentiel de largeur à mi-hauteur $0,8 \mathrm{~nm}$, les autres paramètres restant inchangés, a pour résultat l'obtention

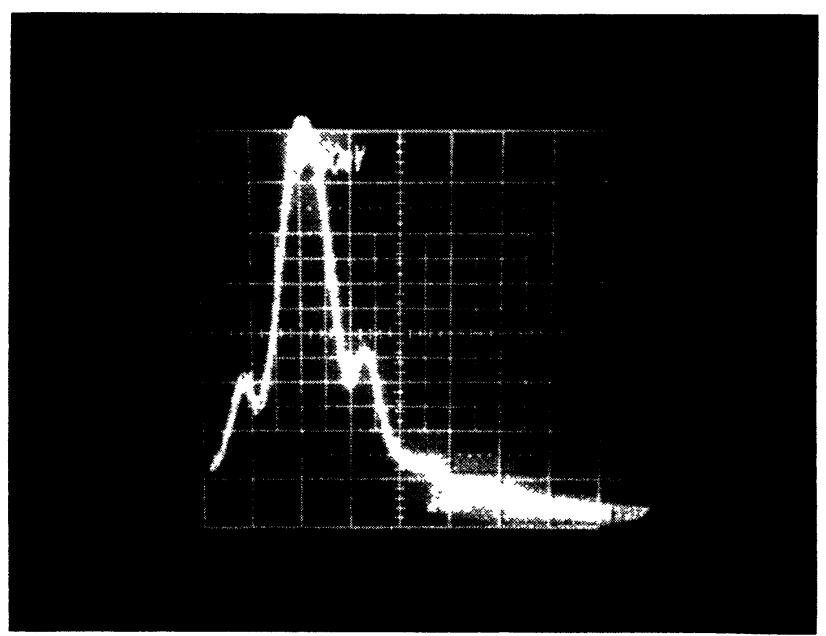

Fig. 9. - Autocorrélation d'une impulsion compressée sur un spectre de $4 \mathrm{~nm}$.

[Autocorrelation trace of a $40 \AA$ bandwidth compressed pulse.] 


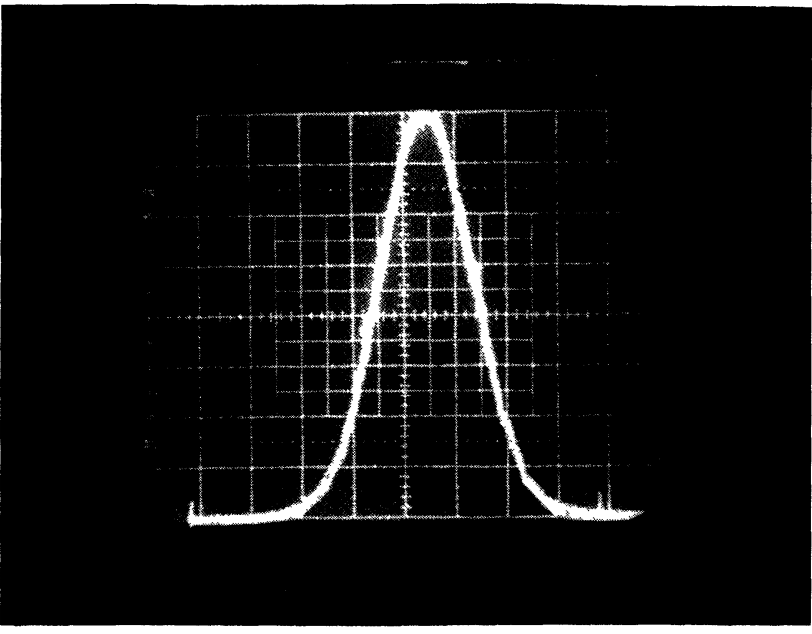

Fig. 10. - Autocorrélation d'une impulsion compressée avec un filtrage spectral de $0,8 \mathrm{~nm}$ à mi-hauteur.

[Autocorrelation trace of a filtered $8 \AA$ bandwidth compressed pulse.]

d'impulsions compressées très proches de la gaussienne théorique, de largeur à mi-hauteur 2 ps (Fig. 10). Les simulations ainsi que les mesures montrent clairement que l'utilisation d'une fibre plus longue afin d'augmenter la durée de l'impulsion possédant un « chirp » n'est pas une solution viable, puisqu'elle a pour effet l'allongement et la déformation de l'impulsion compressée. L'imparfaite compensation de leur fonction de transfert temporelle respective est donc une limitation inhérente de la technique de compression à fibre et à réseaux. Le même problème a été rencontré dans le domaine de la compression d'impulsions femtosecondes. BritoCruz a été capable de le surmonter et de générer des impulsions de 6 fs en utilisant à la fois des réseaux et des prismes pour compenser le « chirp » au premier et au deuxième ordre [12]. Cette solution n'est pas applicable dans notre cas : les prismes ne sont pas suffisamment dispersifs pour que leur utilisation dans le domaine de la picoseconde conduise à des dimensions raisonnables.

Martinez [13] a proposé l'insertion d'un télescope entre les deux réseaux pour générer une dispersion de vitesse de groupe positive. Il devrait alors être possible d'allonger et de compresser l'impulsion avec des systèmes dont les dispersions se compensent parfaitement. Nous avons récemment démontré la validité de cette technique en étirant une impulsion plus de mille fois, puis en la recompressant à sa valeur initiale, ceci en utilisant une combinaison de réseaux pour générer de la dispersion de vitesse de groupe positive et négative [14].

La source utilisée lors de l'expérience était un laser à colorants à verrouillage de modes et collision d'impulsions pompé en synchronisme. Ce laser délivre des impulsions de 80 fs dont la bande passante

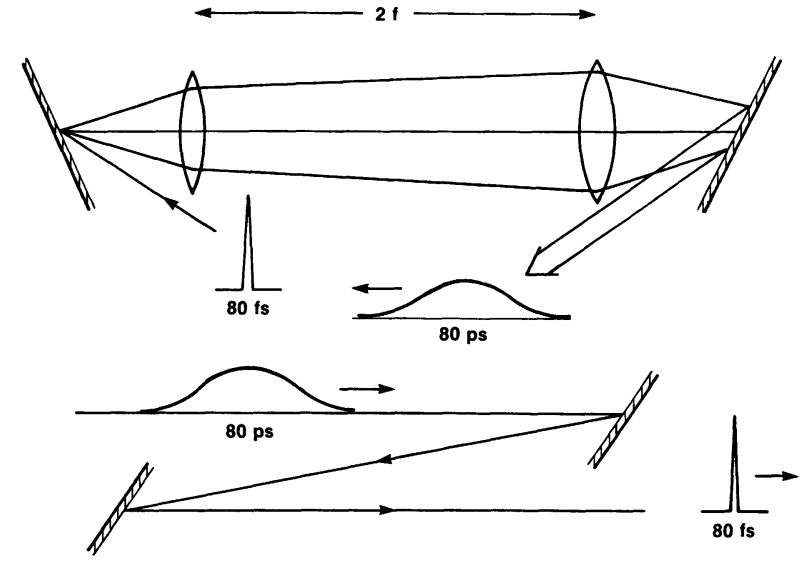

Fig. 11. - Description du système d'étirement-compression.

[Expansion-compression set up.]

est de $4,8 \mathrm{~nm}$ centrée à $617 \mathrm{~nm}$. La configuration expérimentale pour l'allongement et la compression est montrée par la figure 11 . La première partie est composée de deux réseaux holographiques (1 700 traits par mm) situés de part et d'autre d'un télescope de grandissement unitaire et placés entre les plans principaux et les plans focaux dans une configuration anti-parallèle de manière à générer une dispersion de vitesse de groupe positive. Un double passage de l'impulsion à travers ce système conduit à une impulsion d'une durée de 85 ps. Deux réseaux identiques utilisés cette fois dans la configuration parallèle standard ramènent alors l'impulsion à une durée de $90 \mathrm{fs}$ (voir Fig. 12). Cela démontre clairement que l'allongement et la compression se compensent parfaitement.

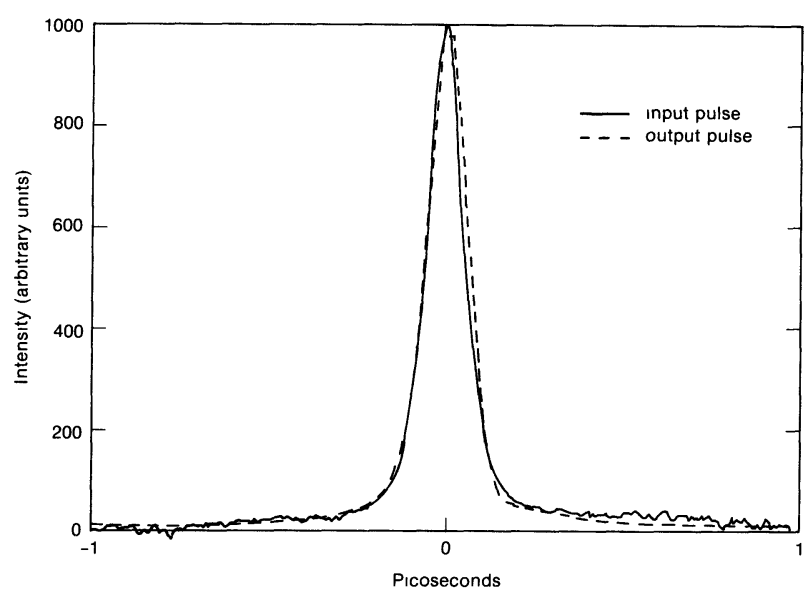

Fig. 12. - Comparaison des traces d'autocorrélation des impulsions à l'entrée et à la sortie du système d'étirementcompression.

[Comparison of the autocorrelation traces of the input and output pulses from the expansion-compression stage.] 
En utilisant cette technique, nous sommes maintenant capables de totalement découpler la durée de l'impulsion compressée de celle de l'impulsion à « chirp ». Il est alors envisageable d'utiliser la technique de compression à double étage de l'oscillateur $\mathrm{Nd}$ : YLF pour générer des impulsions de $100 \mathrm{fs}$. L'allongement ultérieur jusqu'à 500 ps avant amplification permettrait de tirer pleinement avantage de la bande passante du verre au néodyme. L'amplification de ces impulsions jusqu'au niveau du joule, suivie par leur compression, donnerait alors naissance à des puissances instantanées de $1 \mathrm{~J} / 100 \mathrm{fs}=$ $10 \mathrm{TW}$ avec notre système d'amplification actuel.

En conclusion, l'amplification d'impulsions à dérive de fréquence a permis la génération d'impulsions de durée $1 \mathrm{ps}$ au niveau du térawatt. La divergence du faisceau étant 2,2 fois la limite de la diffraction, la brillance de cette source est supérieure à $10^{+18} \mathrm{~W}\left(\mathrm{~cm}^{2} . \mathrm{sr}\right)$, ce qui constitue la brillance la plus élevée jamais reportée. Cette technique est limitée à un rapport de compression de quelques centaines par les termes non linéaires présents dans la fonction de transfert temporelle d'une paire de réseaux. Une amélioration de cette technique a été proposée, basée sur l'utilisation d'une paire de réseaux à la fois pour l'allongement et pour la compression de l'impulsion. Un rapport de compression de 1000 a déjà été démontré, et un facteur de
5000 devrait être possible. Cela signifie que des puissances instantanées de l'ordre de $10 \mathrm{TW}$ seront possibles avec le même étage d'amplification. Encore plus excitant est le fait que la technique d'amplification d'impulsions à dérive de fréquence peut être utilisée averc un amplificateur à verre dopé au néodyme de larges dimensions tel que le Glass Development Laser à Rochester. Un tel système est capable d'amplifier des impulsions de $1 \mathrm{~ns}$ au niveau $\mathrm{du} \mathrm{kJ}$. Ces impulsions, après recompression, pourraient générer une puissance instantanée supérieure au pétawatt.

\section{Remerciements.}

Nous remercions le Laboratoire d'Optique Appliquée, ENSTA, Palaiseau, France, pour le prêt de l'amplificateur de $16 \mathrm{~mm}$. Ce travail a été supporté par l'United State Air Force Office of Scientific Research sous le contrat F49620-87-C-0016 pour l'Ultrafast Optical Electronic Center au Laboratory for Laser Energetics of the University of Rochester et par le Laser Fusion Feasibility Project au Laboratory for Laser Energetics qui a les sponsors suivant : Empire State Electric Energy Research Corporation, General Electric Company, New York State Energy Research and Development Authority, Ontario Hydro et l'University of Rochester.

\section{Bibliographie}

[1] KoEchner, W., Solid-State Laser Engineering (Springer-Verlag) 1976, p. 581.

[2] Schwarzenbach, A. P., Luk, T. S., Mcintyre, I. A., Johann, U., MCPherson, A., Boyer, K. and RHODES, C. K., Subpicosecond $\mathrm{KrF}^{*}$ Excimer-Laser Source, Opt. Lett. 11 (1986) 499.

[3] Deshazer, L. G., Advances in Tunable Solid-State Lasers, Laser Focus 23 (1987) 54-58.

[4] Cook, C. E., Pulse Compression-Key to More Efficient Radar Transmission, Proc. IRE 48 (1960) 310-316.

[5] Strickland, D. and Mourou, G., Compression of Amplified Chirped Optical Pulses, Opt. Commun. 56 (1985) 219-221.

[6] Strickland, D., Maine, P., Bouvier, M., WilLIÁMSON, S. and Mourou, G., Picosecond Pulse Amplification Using Pulse Compression Techniques, Ultrafast Phenomena $V$, Eds G. R. Fleming and A. E. Sigman (SpringerVerlag) 1986 , p. 38-42.

[7] BAdo, P., Bouvier, M. and Coe, J. S., Nd : YLF Mode-Locked Oscillator and Regenerative Amplifier, Opt. Lett. 12 (1987) 319-321.

[8] Nakatsuka, H. and GrischKowsky D., Recompression of Optical Pulses Broadened by Passage
Through Optical Fibers, Opt. Lett. 6 (1981) 1315.

[9] Desbois, J., Gires, F. and Tournois, P., A New Approach to Picosecond Laser Pulse Analysis Shaping and Coding, IEEE J. Quantum. Electron. QE-9 (1973) 213-218.

[10] Janszky, J., Corradi, G. and Gyuzalian, R. N., On a Possibility of Analysing the Temporal Characteristics of Short Light Pulses, Opt. Commun. 23 (1977) 293-298.

[11] MCMullen, J. D., Analysis of Compression of Frequency Chirped Optical Pulses by a Strongly Dispersive Grating Pair, Appl. Opt. 18 (1979) 737-741.

[12] Brito-Cruz, C. H., Fork, R. L. and Shank, C. V., Compression of Optical Pulses to $6 \mathrm{fs}$ Using Cubic Phase Distortion Compensation, paper MD1, CLEO (1987).

[13] Martinez, O. E., Gordon, J. P. and Fork, R. L., Negative Group-Velocity Dispersion Using Refraction, J. Opt. Soc. Am. A 1 (1984) 10031006.

[14] Pessot, M., Maine, P. and Mourou, G., 1000 Times Expansion/Compression of Optical Pulses for Chirped Pulses Amplification, Opt. Commun. to be published. 